DOI: $10.14451 / 1.202 .137$

\title{
ОЦЕНКА И СЕЛЕКЦИЯ РИСКОВ ТОРГОВОГО ПРЕДПРИНИМАТЕЛЬСТВА В УСЛОВИЯХ ПОВЫШЕННОЙ НЕОПРЕДЕЛЁННОСТИ И ЭКОНОМИЧЕСКОЙ НЕСТАБИЛЬНОСТИ
}

\author{
(c) 2021 Кунин Владимир Александрович \\ доктор экономических наук, доцент \\ Санкт-Петербургский университет технологий управления и экономики, Россия, Санкт-Петербург \\ (c) 2021 Михайловский Дмитрий Александрович \\ аспирант \\ Санкт-Петербургский университет технологий управления и экономики, Россия, Санкт-Петербург \\ коммерческий директор $3 \mathrm{AO}$ «Альянс-ГРУПП»
}

В настоящей статье систематизируются методы и показатели оценки предпринимательских рисков. Применительно к торговому предпринимательству показывается необходимость применения проблемно-ориентированных методов оценки рисков, учитывающих отраслевую специфику этого вида предпринимательской деятельности. Предлагается метод 3D сепарации рисков, предназначенный для селекции частных рисков торговых предпринимательских структур с точки зрения достаточности управления потенциальными рисками на основе ценовой компенсации потерь. Предлагается оценивать риски торговых предпринимательских структур трёхкомпонентным вектором рисков, компонентами которого являются вероятность активации риска, ущерб от его реализации и значимость риска, характеризующая относительное изменение покупательской лояльности под воздействием оцениваемого риска. Даются практические рекомендации по определению критериев 3D сепарации рисков на основе использования их трёхкомпонентных векторов для торговых предпринимательских структур разного масштаба. Оценивается влияние стратегических рисков снижения выручки и повышения общих издержек на ключевые показатели результатов и эффективности предпринимательской деятельности. Оцениваются доверительные границы изменения прибыли и рентабельности под воздействием частных рисков в характерных для настоящего времени условиях неопределённости и экономической нестабильности. Исследуется и оценивается влияние масштаба и рентабельности предпринимательских структур на тяжесть последствий от воздействия стратегических рисков.

Ключевые слова: риск, неопределённость, предпринимательская структура, оценка, эффективность.

\section{Актуальность исследования}

Современные условия ведения бизнеса характеризуются высоким уровнем неопределённости и экономической нестабильности. Это связано как с труднопредсказуемыми внешними рисками, так называемыми «чёрными лебедями», так и с внедрением прорывных технологий, способствующих быстрым изменениям окружающего мира, включая, в частности, изменение рыночной конъюнктуры и предпочтений покупателей. Поэтому для торговых предпринимательских структур весьма актуальной становится задача адекватной оценки внешних и внутренних рисков и своевременной разработки, и реализации превентивных мер, нацеленных на их предотвращение или нейтрализацию.

\section{Системный анализ показателей предпри- нимательских рисков}

В научной литературе проблеме оценки предпринимательских рисков и, в частности, рисков торгового предпринимательства посвящён целый пласт научных работ, в которых применительно к рассматриваемым в этих работах видам рисков предлагаются различные методы их оценки.

При проведении оценки рисков необходимо учитывать иерархическую структуру рисков и сложный характер их взаимного влияния. В этой связи следует обратить внимание на то, что различные частные риски в процессе их активации и взаимного влияния друг на друга в конечном итоге порождают два интегральных хозяйствен- 
ных риска: риск снижения выручки и риск повышения общих издержек [1, с.95]. В дальнейшем мы будем называть эти риски стратегическими, а все факторные риски, активация которых приводит к активации стратегических рисков частными рисками.

Детальная классификация существующих показателей уровня предпринимательских рисков проведена в [1, с.83], где выделяется шесть классов показателей основанных на:

- статистических методах оценки;

- оценке характеристик финансово-хозяйственной деятельности;

- оценке силы воздействия рычагов;

- оценке характеристик безопасности;

- экспертной оценке рисков;

- оценке влияния рисков на результаты и эффективность хозяйственной деятельности.

K показателям, основанным на статистических методах оценки, относят показатели, применяемые для оценки рисков финансового инвестирования, а также кредитных и операционных банковских рисков. К этим показателям, в частности, относят VaR, кредитный VaR, $\beta$-коэффициент, дисперсию доходности портфеля и др. [2] Однако для оценки рисков торгового предпринимательства эти показатели неприменимы. Показатели, уровня рисков других групп применимы для оценки рисков торгового предпринимательства, при этом возможности их применения ограничены. Показатели, основанные на оценке характеристик финансово - хозяйственной деятельности, к которым, в частности, относят абсолютные и относительные показатели финансовой устойчивости, платежеспособности и банкротства, применимы для оценки отдельных финансовых рисков, но неприменимы для выявления и оценки факторов и предвестников других предпринимательских рисков, включая стратегические хозяйственные риски снижения выручки и повышения общих издержек. Показатели, основанные на силе воздействия рычагов, к которым относят коэффициенты операционного и финансового левериджа, важны для выявления предвестников и оценки структурного риска, обусловленного несоответствием структуры издержек конъюнктуре рынка сбыта, и риска потери финансовой устойчивости. Показатели, основанные на оценке характеристик безопасности, таких как запас финансовой прочности и коэффициент безопасности, позволяют оценить риск убыточности хозяйственной деятельности предприятия, но не выявляют факторы этого риска и стратегических рисков снижения выручки и повышения общих издержек. Что касается экспертных методов оценки риска, то эти методы применяются наиболее часто, но обоснованность и достоверность таких оценок далеко не всегда достаточна для принятия адекватных управленческих решений, и разработки эффективных мер предотвращения и нейтрализации рисков.

Среди рассмотренных классов показателей следует выделить показатели, основанные на оценке влияния рисков на результаты и эффективность хозяйственной деятельности. К этим показателям, прежде всего, относят показатели EaR и RAROC [3], характеризующие изменение показателей прибыли и рентабельности под воздействием цепочек частных факторных рисков, порождающих стратегические риски. Однако практическая реализация формирования информационной базы оценки и методика реализации методов оценки указанных показателей в этом и других известных источниках раскрыта недостаточна. Достоверная оценка влияния рисков на результаты и эффективность хозяйственной деятельности является ключевым элементом для выработки мер по управлению рисками. Для получения таких оценок кроме методов оценки показателей EaR и RAROC могут использоваться предложенные и развитые в работах Е.Д. Соложенцева [4,5,6] логико-вероятностные методы или предложенный в [7] метод оценки предельных величин рисков на основе построения треугольника Паскаля. Однако реализация указанных методов затруднена как высокими требованиями к уровню профессиональной и, в частности, математической подготовки специалистов, занимающихся управлением рисками, так и сложностями формирования информационной базы, необходимой для оценки рисков этими методами. Особенно остры эти проблемы для малых и средних предприятий, не имеющих специального штата для решения задач риск - менеджмента. Кроме того, характерный для настоящего времени, высокий уровень неопределённости снижает достоверность оценок, полученных на основе априорных вероятностных параметров рисков, используемых при практической реализации этих методов [8-12].

Из проведённого анализа вытекает необ- 
ходимость разработки проблемно ориентированных, удобных в практическом применении методов оценки частных и стратегических хозяйственных рисков торговых предпринимательских структур и оценки влияния этих рисков на результаты и эффективность хозяйственной деятельности.

Оценка и селекция рисков методом 3D ceпарации

В процессе управления рисками можно выделить 3 стадии: предрисковую стадию, стадию активации риска и стадию последствий. Как отмечалось выше, активация стратегического риска повышения издержек порождает ущерб, который проявляется в уменьшении чистого денежного потока и снижении показателей прибыли и рентабельности торговой предпринимательской структуры. Поэтому для компенсации этого ущерба целесообразно применить метод ценовой компенсации потерь [13], являющийся эффективным инструментом управления последствиями активации риска повышения общих издержек. Отличительной особенностью данного метода является то, что в процессе ценообразования потери от воздействия частных рисков, порождающих повышение общих издержек, изначально не закладываются в цену товара, а компенсируются в последующем путем распределения ценовой компенсации ущерба от риска на определенный достаточно длительный (год и более) период времени. При этом компенсационное увеличение цены должно быть незначительным и практически незаметным для покупателя, чтобы не активировать риски снижения покупательского спроса на данный товар и снижения выручки. Такой подход позволяет переложить дополнительные расходы на плечи потребителя, сохранив при этом ликвидность товарной линейки и покупательскую лояльность на прежнем уровне. Вместе с тем необходимо отметить, что не для всех частных рисков, являющихся факторными рисками по отношению к риску повышения общих издержек, удастся добиться компенсации ущерба только на основе ценовой компенсации.

В целях обеспечения компенсации или хотя бы эффективного уменьшения негативных последствий всех потенциальных частных рисков в настоящей работе предлагается проводить их предварительную селекцию на основе оценки методом 3D сепарации рисков. В рамках этого метода на предрисковой стадии риск-менеджмента осуществляется селекция рисков по приемлемости применения, для управления последствиями их активации, метода ценовой компенсации потерь. Если по результатам 3D ceпарации риск относится к приемлемым, то для компенсации ущерба от его активации достаточно использовать метод компенсации потерь. Если же риск отнесён к неприемлемым, то для компенсации или снижении ущерба требуется реализация дополнительных мер, нацеленных на снижение вероятности реализации риска, ущерба от риска и значимости риска для снижения лояльности покупателей.

Структурная схема реализации метода 3D ceпарации рисков для оценки рисков и разработки адекватных мер управления рисками приведена на рис. 1.

В процессе оценки рисков определяются следующие показатели:

1. Точка 0 (Т0). Это величина равная запланированным ежемесячным расходам торгового предприятия и является базовой для дальнейшей оценки рисков, нормируемых относительно этой величины.

2. Вероятность активации риска $\left(\mathrm{P}_{\mathrm{aK}}\right)$, Оценка осуществляется в количественном выражении.

3. Ущерб $\left(\mathbf{B}_{\mathbf{p}}\right)$ - величина возможного ущерба от единичного случая воздействия риска. Применяется для расчета и оценки общей величины ущерба рисков оказывающих многоразовое воздействие. На этапе разработки превентивных мероприятий данный показатель необходим для оценки требуемых ресурсов для снижения или компенсации возможного ущерба (резервы, кредиты и т.д.). Ущерб и вероятность активации риска могут, в частности, определятся методами экспертных оценок.

4. Значимость риска (3н). Представляет собой величину, характеризующую возможное относительное изменение показателя покупательской ликвидности товарной линейки (ППЛ под воздействием риска. Показатель покупательской ликвидности товарной линейки отражает уровень привлекательности товарного ассортимента с точки зрения покупателя, то есть характеризует ликвидность товара, имеющего определенные качественные и функциональные свойства и установленную цену на отдельно взятом локальном рынке. ППл определяется по 


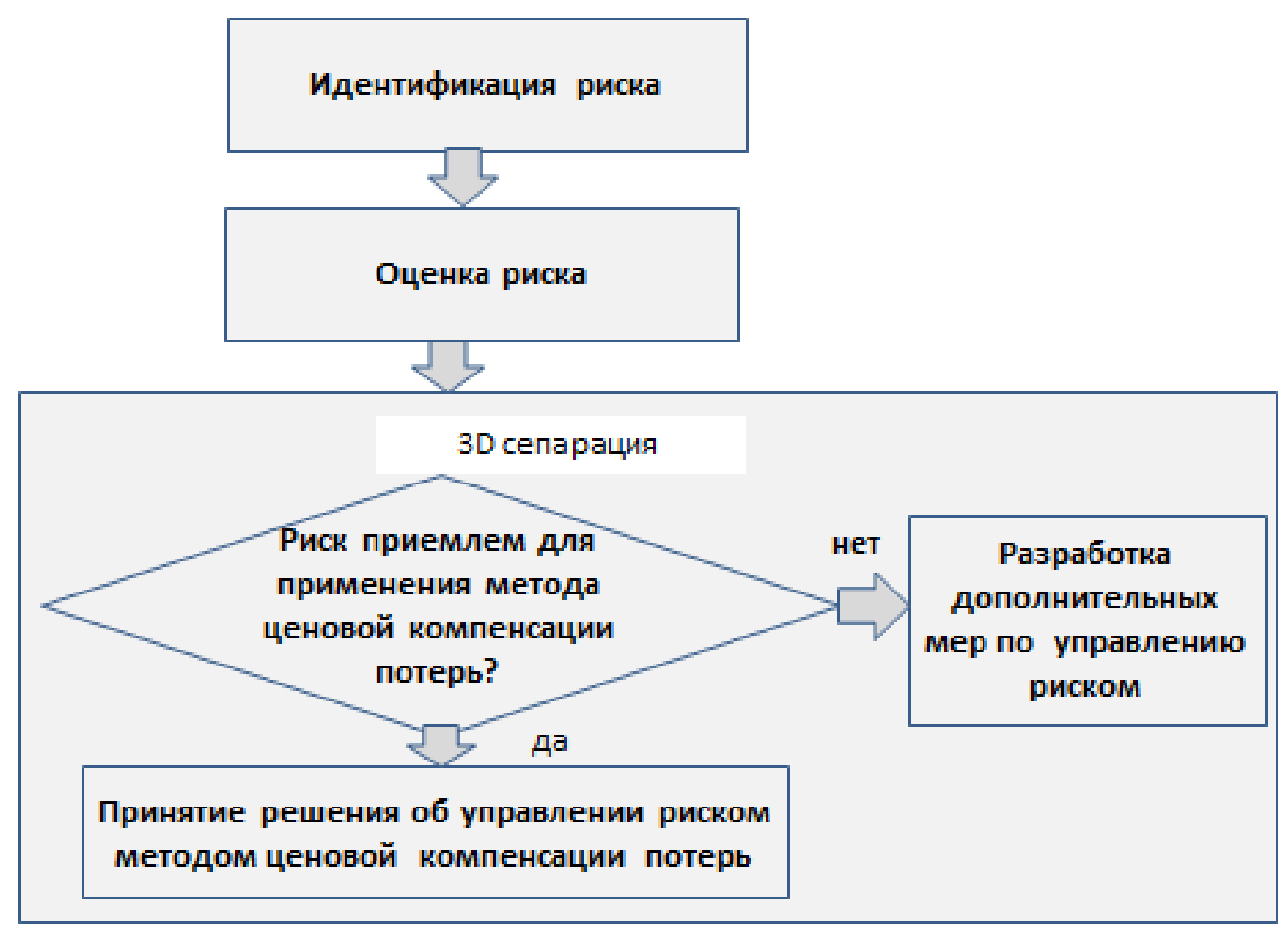

Puc. 1. Структурная схема селекции и оценки рисков методом 3D сепарации

формуле [14]

$$
\text { ППЛ }=\frac{K \cdot \sum_{n=1}^{m} K_{n}}{m}
$$

В формуле (11) использованы следующие обозначения:

$K$ - показатель качества товара, определяемый по формуле

$$
K=\frac{K_{o в}}{K_{n y}+K_{6 p}}
$$

$K_{\text {ов }}$ - показатель общего восприятия товара покупателем;

$K_{n y}-$ показатель наличия повреждений упаковки товара;

$K_{б р}-$ показатель наличия брака или скрытых дефектов;

$\mathrm{m}$ - количество частных показателей, учитываемых при определении ППЛ $(\mathrm{m} \geqslant 6)$;

$K_{n}$ - показатели, отражающие различные характеристики товара, в частности,

$K_{1}$ - показатель функциональности товара;

$K_{2}$ - показатель дизайна (внешнего вида) товара;

$K_{3}-$ показатель приемлемости цены товара;
$K_{4}$ - показатель покупательской лояльности к бренду/торговой марке и т.д.

Показатель брака определяется персоналом торгового предприятия, как доля выявленных бракованных единиц товара в общем количестве данного товара поступившего на торговое предприятие за достаточно длительный интервал времени. Остальные показатели определяются по результатам опроса покупателей и статистической обработки результатов этого опроса.

Полная информация о лояльности покупателей и влиянии риска на лояльность формируется при трёхуровневой оценке показателя покупательской ликвидности. На первом уровне оценивается ППЛ по каждому виду товара, на втором - по каждой товарной линейке, а на третьем - по всему товарному ассортименту торгового предприятия. Оценку ППЛ по товарной линейке целесообразно производить взвешенным усреднением этих показателей по отдельным товарам из линейки. При этом в качестве весовых коэффициентов могут использоваться доли каждого из товаров товарной линейки. Аналогично, взвешенным суммированием показателей покупательской ликвидности по товарным линейкам оценивается значение этого показателя по всему товарному ассортименту. При этом в качестве весовых коэффициентов 
можно использовать доли различных товарных линеек во всём ассортименте.

По результатам оценки показателей риска в настоящей работе предлагается формировать трёхкомпонентный вектор $\bar{R}=\left(\mathrm{P}_{a \kappa}, \mathrm{B}_{p}, 3_{\mu}\right)$, составляющими которого являются значения вероятности активации риска, ущерба и значимости риска. В целях наглядности представления информации, составляющие трёхкомпонентного вектора риска целесообразно проградуировать и изобразить выделенные градации в виде цветной шкалы, характеризующей уровень риска по каждой из компонент. Пример возможной шкалы компонент трёхмерного вектора риска представлен на рис. 2.

Полученная шкала компонент вектора риска даёт возможность проводить дальнейшую сепарацию и выявление рисков, для которых ценовая компенсация потерь недостаточна, различными способами.

Наиболее простой способ сепарации заключается в эмпирическом формировании критерия сепарации рисков на основе накопленного опыта управления рисками и представлений о вкладе каждой из компонент в интегральный уровень риска и тяжесть последствий его активации для предприятия. Так, например, можно считать приемлемым риск, для которого все 3 компоненты либо находятся в зелёных зонах, либо в этих зонах находятся все компоненты кроме вероятности активации риска, для которой допускается попадание в жёлтую зону. Следует отметить, что дополнительную помощь в формировании критерия сепарации рисков может оказать визуализация трёхкомпонентных векторов рисков на дашбордах. Этот способ сепарации целесообразно применять для малых и средних торговых предприятий, имеющих весыма ограниченные ресурсы для внедрения в практику риск - менеджмента сложных цифровых технологий, включая технологии искусственного интеллекта.

Для крупных торговых предприятий, обладающих более широкими возможностями, для формирования критериев сепарации рисков целесообразно использовать методы машинного обучения с использованием нейронных сетей. На основе применения методов машинного

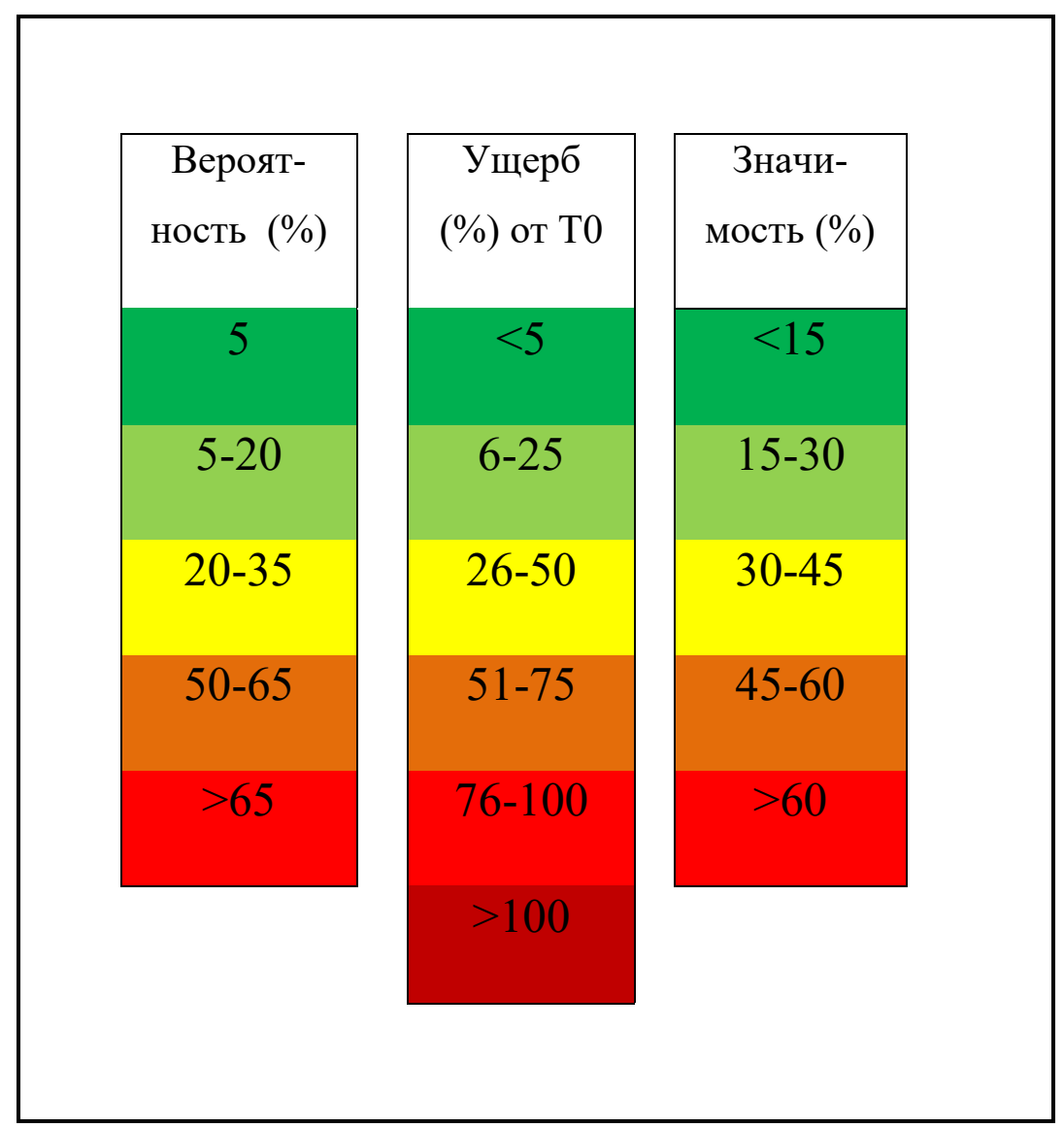

Puс. 2. Шкала компонент вектора риска 
обучения в трёхмерном пространстве строится поверхность, разделяющая приемлемые и неприемлемые риски. Применение этих методов позволит, используя постоянно накапливающуюся базу данных об активировавшихся рисках и тяжести их последствий совершенствовать критерии сепарации и повышать эффективность применения метода 3D сепарации рисков.

Оценка влияния стратегических хозяйственных рисков на результаты и эффективность хозяйственной деятельности

Применительно к торговым предпринимательским структурам в качестве ключевых показателей результатов и эффективности предпринимательской будем соответственно рассматривать прибыль от продаж и рентабельность продаж. Количественные оценки относительного изменения этих показателей под воздействием стратегических хозяйственных рисков снижения выручки и повышения общих издержек получены в [15]. В этой работе получены зависимости между абсолютными и относительными изменениями выручки, общих издержек, прибыли и рентабельности продаж, которые имеют вид:

$$
\begin{aligned}
& \Delta \Pi_{\Pi P}=\Pi_{n p 2}-\Pi_{n p 1}=\Delta B H+\Delta U_{0}, \\
& \delta \Pi_{\Pi P}=\frac{\Pi_{n p 2}-\Pi_{n p 1}}{\Pi_{n p}}=\frac{B H \cdot \delta B H-h_{O} \cdot \delta \Pi_{O}}{\Pi_{n p}} \\
& \Delta P_{\Pi P}=P_{n p 2}-P_{n p 1}=\frac{\kappa_{U} \cdot(\delta B H+\delta H)}{1+\delta B H} \\
& \delta P_{\Pi P}=\frac{\kappa_{U} \cdot(\delta B H+\delta H)}{P_{n p} \cdot(1+\delta B H)}
\end{aligned}
$$

В формулах (1)-(4) использованы следующие обозначения

$\Delta B H, \quad \Delta \Lambda_{0}, \Delta \Pi_{n p}, \quad \Delta P_{n p}-$ соответственно предельные абсолютные отклонения выручки, общих издержек, прибыли от продаж и рентабельности продаж под воздействием предпринимательских рисков, оцениваемые с заданным уровнем доверия $\alpha$;

$\delta B H, \delta И_{0}, \delta \Pi_{n p}, \delta P_{n p}-$ соответственно предельные относительные отклонения выручки, общих издержек, прибыли от продаж и рентабельности продаж под воздействием предпри- нимательских рисков, оцениваемые с заданным уровнем доверия $\alpha$;

$\kappa_{И}=h_{0} / B H-$ коэффициент уровня издержек, характеризуемый долей общих издержек в выручке.

Доверительный интервал анализируемого показателя прибыли или рентабельности определяется величиной [15]:

$$
\Delta \rho=\left[p_{\text {ож }}-\kappa \cdot \sigma_{p} \div p_{\text {ож }}+\kappa \cdot \sigma_{p}\right]
$$

где

$p_{\text {ож }}$ - ожидаемое значение анализируемого показателя $p$;

$\kappa$ - показатель «ширины» доверительного интервала, определяемый уровнем доверия и функциональным видом плотности вероятностей анализируемого показателя;

$\sigma_{p}$ - среднеквадратичное отклонение анализируемого показателя $p$, определяемое равенством

$$
\sigma=\frac{|\Delta p|}{2 \kappa}
$$

где $\left|\Delta_{p}\right|$ - абсолютная величина предельного абсолютного отклонения анализируемого показателя $p$.

Ширина интервала (5) зависит от уровня неопределённости. Чем шире интервал - тем выше уровень неопределённости и, как следствие, выше риск недопустимого снижения анализируемого показателя прибыли или рентабельности под воздействием рисков.

При выполнении условий действия закона больших чисел, когда показатель $p$ определяется воздействием большого числа факторов, среди которых нет доминирующих, можно допустить, что этот показатель подчиняется нормальному закону распределения и, например, при уровне доверия $\alpha=0,99$ параметр «k» принимает значение равное 2,62.

Проведём ситуационный анализ влияния стратегических хозяйственных рисков на прибыль от продаж и рентабельность продаж.

Ситуация 1: риск повышения общих издержек пренебрежимо мал, т.е. $\delta И_{O} \rightarrow 0$.

В этой ситуации

$$
\begin{aligned}
& \delta \Pi_{\Pi P}=\frac{B H \cdot \delta B H-H_{O} \cdot \delta H_{O}}{\Pi_{n p}} \rightarrow \frac{B H}{\Pi_{n p}} \cdot \delta B H=\frac{\delta B H}{P_{n p}} \\
& \delta P_{\Pi P}=\frac{\kappa_{h} \cdot\left(\delta B H+\delta H_{O}\right)}{P_{n p} \cdot(1+\delta B H)} \rightarrow \frac{\kappa_{H} \cdot \delta B H}{P_{n p} \cdot(1+\delta B H)}
\end{aligned}
$$


Отсюда следует, что для низкорентабельных торговых предприятий, что особенно характерно для торговых предприятий малого бизнеса, т.е. при малых значениях $P_{n p}=1$ относительные снижения прибыли от продаж и рентабельности продаж велики даже при сравнительно небольших снижениях выручки под воздействием рисков.

Ситуация 2: риск снижения выручки пренебрежимо мал, т.е. $\delta B H \rightarrow 0$.

В этой ситуации

$\delta \Pi_{\Pi P}=\frac{B H \cdot \delta B H-h_{O} \cdot \delta h_{O}}{\Pi_{n p}} \rightarrow \frac{h_{O}}{\Pi_{n p}} \cdot \delta h_{O}=\frac{\Delta U_{O}}{\Pi_{n p}}$

$\delta P_{\Pi P}=\frac{\kappa_{U} \cdot\left(\delta B H+\delta H_{O}\right)}{P_{n p} \cdot(1+\delta B H)} \rightarrow \frac{\kappa_{h} \cdot \delta h_{O}}{P_{n p}}$

Из полученных формул следует, что для малоприбыльных предприятий, характеризуемых низкими значениями прибыли от продаж, сопоставимыми с величиной абсолютного изменения общих издержек, относительное изменение прибыли от продаж под воздействием риска повышения общих издержек велико, может достигать и даже превышать величину 100\%. У таких предприятий даже сравнительно небольшое увеличение общих издержек под воздействием рисков может привести к убыточной деятельности. Относительное снижение рентабельности продаж под воздействием риска повышения общих издержек для низкорентабельных предприятий также велико даже при сравнительно небольшом повышении общих издержек.

\section{Заключение}

Из проведённого анализа следует, что низкорентабельные торговые предприятия и, в частности, многие предприятия малого бизнеса весьма чувствительны к рискам снижения выручки и повышения общих издержек. Тяжесть воздействия этих рисков на малые предприятия усугубляется непрофессиональным управлением рисками из-за недостаточной квалификации кадров и отсутствия необходимых инвестиционных ресурсов.

Однако применение проблемно - ориентированных методов оценки рисков, учитывающих отраслевую специфику наряду с предлагаемым методом 3D сепарации рисков, снижает, в процессе риск - менеджмента, зависимость предпринимательских структур от перечисленных выше ресурсов. При этом, за счет системного анализа показателей предпринимательских рисков, ориентированного на оценку изменений потребительских предпочтений на локальном рынке, обеспечивается оперативная оценка рисков товарно-договорной группы и их нейтрализация.

Предложенная оценка влияния стратегических рисков снижения выручки и повышения общих издержек на ключевые показатели результатов и эффективности предпринимательской деятельности позволяет обеспечить адекватное и своевременное управление частными рисками на предрисковой стадии риск-менеджмента. При этом рекомендованные компенсационные методы управления рисками создают условия для нейтрализации стратегических рисков на стадии последствий.

\section{Библиографический список}

1. Кунин В.А. Управление рисками промышленного предпринимательства (теория, методология, практика).СПб.: Издательство Санкт-Петербургской академии управления и экономики. 2011. - 184c.

2. Энциклопедия финансового риск - менеджмента /Под ред. Лобанова и Чугунова - 2-е издание, М.: - Альпина Бизнес Букс, 2006.- 878 с.

3. Томас Бартон, Уильям Шенкир. Риск - менеджмент. Практика ведущих компаний.- М.: Вильямс ИД, 2008. 208c.

4. Соложенцев Е. Д. Сценарное логико-вероятностное управление риском в бизнесе и технике. 2-е изд. СПб.: Бизнес - пресса, 2006.- 560 с.

5. Соложенцев Е. Д., Махутов Н.А. Логико-вероятностные модели риска в многокомпонентных системах с группами несовместных событий для задач классификации инвестирования эффективности и менеджмента //Проблемы безопасности и чрезвычайных ситуаций. - 2006. -№ 3.-. С.30-52.

6. Соложенцев Е.Д. Управление риском и эффективностью в экономике: Логико- вероятностный подход. СПб.: Издательство СПбГУ, 2009.-259с..

7. Кунин В. А., Фоминых А. И. Концепция управления рисками сбыта в условиях трансформации конкурентной среды/Экономика и управление.-2014. - № 9(107). - С. 38-47. 
8. Галеева И. Н., Сердарова М. С., Егоров Д. А. Анализ рисков инновационного развития сферы информационных услуг//Экономика и предпринимательство. - 2020. - № 12(125). - С.1267-1271.

9. Данилина Я. В., Рыбачук М. А. Системные эффекты и риски цифровой экономики: Анализ с позиции системной экономической теории/Экономическая наука современной России. - 2019. - № 3 (86). - С. $119-138$.

10. Темиргалиева Р. Р., Гришин И. Ю., Коротицкая В.В. Риски в условиях развития цифровой экономики России//Научно-методический электронный журнал Концепт. - 2019. - № 6. - С. 240-246.

11. Талдонова С.С. Становление и развитие цифровой экономики в мире//Экономика и предпринимательство. - 2020. - № 12 (125). - С. 177-180.

12. Кот М.К., Белозерова О.А., Шпанагель Ф.Ф. Преимущества и риски цифровой экономики: Правовой и экономический аспекты.//Актуальные проблемы правоведения. - 2018. - № 4 (60). - С. 6-11.

13. Михайловский Д.А. Компенсационный метод управления последствиями активации предпринимательских рисков//Проблемы современной экономики. - 2020. - № 3(75). - С.107-109.

14. Михайловский Д. А. Управление рисками снижения покупательской ликвидности товарной линейки торговых предпринимательских структур//Проблемы современной экономики. - 2020. - № 1.(73). - С. 71-74.

15. Кунин В.А. Кластеризация предпринимательских решений в условиях неопределённости.//Проблемы современной экономики. - 2016.- № 2.С.117-120. 